\title{
Second- and Third-Order Signal Predistortion for Nonlinear Distortion Suppression in a TWT
}

\author{
Aarti Singh, John E. Scharer, Senior Member, IEEE, John H. Booske, Senior Member, IEEE, and John G. Wöhlbier
}

\begin{abstract}
The nonlinearity inherent in the traveling wave tube (TWT) amplifier distorts amplified signals and results in reduced efficiency and bandwidth, limiting its use in communication and electronic countermeasure applications. Signal predistortion is an effective technique for suppressing nonlinear distortion in TWTs that provides high suppression and requires simple circuits for implementation. While conventional predistortion linearizers are based on third-order intermodulation (3IM) injection, this paper proposes using second-order (second-harmonic) signal injection in predistortion circuits. A detailed experimental investigation and comparison of second-order versus third-order signal predistortion is presented. It is observed that both schemes result in suppression of up to $30 \mathrm{~dB}(55 \mathrm{dBc})$ for the $3 \mathrm{IM}$ distortion products. However, experimental results indicate that second-harmonic signal injection performs better than 3IM in suppressing higher order products. The paper also investigates spatial evolution of the wave spectrum along the TWT axis with and without injection, and sensitivity of the suppression to injected signals amplitude, phase and the fundamental frequency.
\end{abstract}

Index Terms-Distortion suppression, harmonic injection, predistortion, signal injection, traveling wave tubes (TWTs).

\section{INTRODUCTION}

$\mathbf{T}$ RAVELING wave tubes (TWTs) are microwave amplifiers widely used in satellite communications and electronic countermeasures (ECM). The high output power and high efficiency of these devices makes them ideally suited as broadcast amplifiers in satellite communications. For ECM applications, high-power enables jamming of enemy radars and wide bandwidth allows spoofing by pseudo-noise and frequency-hopping spread-spectrum techniques.

While TWT amplifiers have high-power capabilities and broad gain-bandwidths, the nonlinearity of these devices results in amplitude, phase and spectral distortion. Nonlinear distortion products appear as harmonics $(f, 2 f, 3 f \ldots)$ and, for multicarrier operation, intermodulation products $\left(m f_{a} \pm n f_{b} \pm \cdots\right)$ at the output of the amplifier. Since these distortion products become more significant as the tube is driven close to saturation to achieve maximum output power, the usable bandwidth of the amplifier and the fundamental efficiency is degraded. This

Manuscript received July 21, 2004; revised November 15, 2004. This work was supported in part by AFOSR under Grant 49620-00-1-0088 and by DUSD (S\&T) under the Innovative Microwave Vacuum Electronics Multidisciplinary University Research Initiative (MURI) program, managed by the U.S. Air Force Office of Scientific Research under Grant F49620-99-1-0297. The review of this paper was arranged by Editor P. Waller.

A. Singh, J. E. Scharer, and J. H. Booske are with the Department of Electrical Engineering, University of Wisconsin, Madison, WI USA 53706.

J. G. Wöhlbier is with the Los Alamos National Laboratory, Los Alamos, NM 87545 USA.

Digital Object Identifier 10.1109/TED.2005.845794 spectral growth also causes spill-over of one channel's signal into adjacent channels (adjacent channel interference) as well as distorts the signal within a channel causing intersymbol interference. Thus, suppression of harmonics and intermodulation frequencies in the output spectra of TWT amplifiers is desirable for reliable and high data-rate multicarrier communication.

One way to limit the nonlinear distortion is to operate the TWT a few dB "backed off" from saturation. However, this compromises output power and device efficiency. Numerous techniques have been pursued for reducing distortion and improving linearity of microwave power amplifiers, in general, and TWT amplifiers, in particular. These techniques include conventional third-order based predistortion [1]-[4], feedforward [5], feedback [6], helix voltage modulation [7] and linearization using nonlinear components (LINC) [8], [9]. Each of these techniques faces its own inherent challenges. For example, predistortion requires a good estimate of the amplifiers amplitude and phase characteristics over the device bandwidth and tuning to match these parameters. Also, conventional third-order based predistortion equalizers designed to suppress lower-order intermodulation products, are known to aggravate higher order distortion products. Feedback, feed-forward and helix voltage modulation schemes face bandwidth limitations due to delays in lines and feedback loops, as well as stability and drift problems. LINC on the other hand requires matched modulators and amplifiers. Further, generation of constant amplitude signal components via highly linear signal separation and low-loss, high-isolation power combining are challenging issues. This motivates continued investigation and further insights into linearization techniques that are simple, broadband and effective.

This paper proposes second-order based signal predistortion, and evaluates and contrasts the performance of second-harmonic and third-order intermodulation (3IM) injection based predistortion schemes for suppressing nonlinear distortion in TWTs. Signal predistortion techniques involve conditioning the output spectra of an amplifier by injecting signal(s) of proper amplitude and phase to cancel the nonlinear distortion product(s). While second-order, i.e., second harmonic $(2 f)$ "signal injection" techniques have been most commonly considered for harmonic suppression to improve fundamental $(f)$ output power and efficiency in ECM applications [10], [11], they have been proposed as a "signal predistortion" method to suppress third-order intermodulation (3IM) distortion products [19].

On the other hand, the conventional signal predistortion circuit is designed to generate 3IM distortion products and inject them along with the primary message signal. The amplitudes and phases of the "injected" 3IM products are indirectly 
adjusted by adjusting the transfer characteristics of the predistortion circuit to invert or equalize the measured transfer characteristics (amplitude-amplitude and amplitude-phase) of the nonlinear amplifier. However, analog predistortion linearizer techniques are often not thought of as being implicitly equivalent to 3IM signal injection techniques. As a result, a direct comparison and evaluation of the full range of possibilities for second-harmonic versus 3IM signal injection/predistortion techniques has not previously been conducted. This is the underlying motivation for this paper. It should be noted that both techniques can be implemented with relatively straightforward circuitry. Third-order techniques, for example, can be implemented using either analog predistortion circuit based on two anti-parallel diodes or a field effect transistor (FET) with its linear component cancelled. Second-order signal injection or predistortion techniques can be implemented with a single frequency doubler even for modulated carriers.

This paper presents a detailed experimental investigation of second-harmonic injection and 3IM injection schemes for distortion suppression in TWTs. In order to keep the experimental conditions as similar as possible, we have chosen to experimentally study the physics and effectiveness of both secondand third-order predistortion using similar direct signal injection configurations. In this way, we not only provide a direct comparison of second and third-order predistortion approaches, but also provide a conceptual framework that allows better understanding of the underlying physical mechanisms by which either technique operates within the TWT.

While many signal injection schemes have been proposed for more narrowband solid-state amplifiers, their application to TWTs has not been completely explored and understood. Traditionally, second-harmonic injection has been applied to TWTs for fundamental power output and efficiency enhancement [10], [11]. Initial attempts to explain the physical mechanism responsible for suppression by signal injection are presented in [11] and [12] based primarily on intuition. Second harmonic injection has been studied numerically by Dionne [13] and Datta et al. [15] using nonlinear TWT models. An improved understanding is presented in [16]-[18] based on the frequency-domain nonlinear TWT model S-MUSE. This model gives a modal decomposition of components in the output spectrum and presents a clear picture of the interaction between driven and nonlinear modes at a particular frequency leading to suppression. Suppression of third-order intermodulation or 3IM $\left(2 f_{a}-f_{b}, 2 f_{b}-f_{a}\right)$ products by harmonic injection in TWTs was first investigated by Sauseng et al. [19] who observed a 6-7 dB reduction in the upper and lower 3IMs $\left(2 f_{b}-f_{a}\right.$ and $2 f_{a}-f_{b}$, respectively, assuming $f_{b}>f_{a}$ ) at saturation. More recently, 3IM suppression of up to $24 \mathrm{~dB}$ has been reported by our group [20]. Second-harmonic injection to control 3IM has also been studied analytically [17] and numerically [14], [17].

The experimental setup selected for this study achieves more precise and stable phase selectivity than the prior direct injection experiments, thus resulting in higher suppression than previously reported. Further, we demonstrate that simultaneous suppression of both upper and lower 3IMs in two-tone carrier experiments is possible with a similar high level of reduction. The paper also presents measurements of spatial evolution of the wave spectrum with and without injection along the tube axis, using the XWING TWT that is unique in having sensors located along the tube axis. The sensitivity of the scheme to injected amplitude and phase, as well as the effective bandwidth of suppression is also explored.

The organization of this paper is as follows. Section II presents a brief discussion of the physical mechanism responsible for suppression by signal injection as proposed in [17] and [18]. Section III discusses the experimental device and setup used for performing the signal injection experiments. The two signal injection techniques investigated, second-harmonic injection and 3IM injection, are discussed in Section IV. Section V presents the spatial evolution of the wave spectrum along the axis of the tube with and without injection, while Sections VI and VII investigate the sensitivity of the signal injection schemes to an injected signal's amplitude and phase, and the effective bandwidth of suppression. Section VIII provides a comparison of the two signal injection schemes based on the experimental observations and summarizes the results of the paper.

\section{Physics OF Signal InJeCtion}

Earlier intuitive notions [11], [12] regarding harmonic injection suggested that the mechanism of distortion suppression by signal injection is the destructive interference of the injected signal with the nonlinearly generated distortion product. Simulations using nonlinear codes [13]-[15] have predicted suppression by harmonic injection, for example, but the models used were unable to describe the waveforms in terms of injected and nonlinearly generated components. A comprehensive insight to the physics of signal injection in TWTs was given recently by Wöhlbier et al. [16], [17] based on the analytically solvable nonlinear spectral TWT model S-MUSE [16], [21]. According to S-MUSE, the voltage solution at a frequency $f_{\ell}$ consists of a sum of complex exponential modes representing the dominant waves

$$
\begin{aligned}
V_{\ell}(z, t)= & \left\{A^{\mathrm{dr}} e^{\left(\mu^{\mathrm{dr}}+i \kappa^{\mathrm{dr}}\right) z}\right. \\
& \left.+\sum_{q} A^{\mathrm{n}[[q]} e^{\left(\mu^{\mathrm{n}[q]}+i \kappa^{\mathrm{n}[q]}\right) z}\right\} e^{i 2 \pi f_{\ell}\left(\left(z / u_{0}\right)-t\right)}
\end{aligned}
$$

where $u_{0}$ is the dc beam velocity, the superscript "dr" refers to the driven mode, the superscript " $\mathrm{nl}[q]$ " refers to nonlinearly generated modes of order $q$ formed by beating of two or more frequencies, and $A^{\mathrm{dr}, \mathrm{nl}[q]}$ are the complex amplitudes. Without injection, a signal at frequency $f_{\ell}$ consists of all nonlinearly generated terms formed at that frequency by beating of the fundamentals; for two fundamental frequencies $\left(f_{a}, f_{b}\right)$, these terms are comprised of all $|m|+|n|$ order products for which $m f_{a}+$ $n f_{b}=f_{\ell}$, where $f_{\ell}$ is the frequency of interest. An injected signal can either be represented by a driven mode (if the injected signal has frequency $f_{\ell}$ ) or can result in a nonlinearly generated mode formed by beating of the injected signal with frequencies present in the system without injection. Thus it is the destructive interference of the injected mode (either "driven" or nonlinearly generated with the injected signal) and natural 
nonlinearly generated modes that results in suppression of the natural mode at the TWT output. As predicted in [22] and experimentally verified in [18], the modes have different growth rates $\left(\mu^{\mathrm{dr}}, \mu^{\mathrm{n}[q]}\right)$ and different wavelengths $\left(\beta^{\mathrm{dr}}=\kappa^{\mathrm{dr}}+2 \pi f / u_{0}\right.$, $\left.\beta^{\mathrm{nl}}=\kappa^{\mathrm{nl}}+2 \pi f / u_{0}\right)$. Hence suppression can be achieved at a single axial location along the TWT, typically chosen to be the output.

\section{EXPERIMENTAL SETUP}

The experimental TWT used in this paper is a custom-modified helix research TWT, named experimental Wisconsin Northrup Grumman (XWING). The XWING TWT [23] is a low-voltage $(2.9 \mathrm{kV})$, high space-charge TWT with a broadband gain $(1.5-6 \mathrm{GHz})$ and a single sever to prevent reflections and backward wave oscillations. The TWT is unique in having sensors along the helix that enable study of the spatial evolution of the microwave signal and its spectrum as it is amplified along the device. These sensors are coupled capacitively to the helix at less than $-40 \mathrm{~dB}$ to avoid significant perturbation of the circuit fields.

Fig. 1 shows block diagrams of the experimental setup used for second-harmonic and 3IM signal injection measurements. The two fundamental signals $f_{a}$ at 1.90 and $f_{b}$ at $1.95 \mathrm{GHz}$ are generated using Agilent 83623B signal generators. The generators are frequency locked by sharing a $10 \mathrm{MHz}$ reference to minimize frequency jitter. The harmonic signal $2 f_{b}$ at $3.90 \mathrm{GHz}$ is generated from the fundamental $f_{b}$ using a frequency doubler. For 3IM injection, the 3IM $2 f_{b}-f_{a}$ at $2.00 \mathrm{GHz}$ is generated by mixing the harmonic $2 f_{b}$ and the fundamental $f_{a}$. The frequency doubler is capable of doubling input frequencies from $1.5-5 \mathrm{GHz}$ and avoids any phase drifts between the fundamental and second harmonic or 3IM frequencies thus resulting in higher suppression than reported in [20].

Power levels on the Agilent generators are set to meet the optimum input power specifications of the frequency doubler and the mixer, and minimize spurious frequencies at the output of these nonlinear devices to less than $-20 \mathrm{~dB}$ below the desired signal. The harmonic and 3IM inputs to the TWT are adjusted in amplitude by a series of two attenuators; a coarse 1-dB step attenuator, followed by a multiturn dial fine attenuator. The phase is adjusted using two Narda $1-5-\mathrm{GHz}$ phase shifters with $0.2^{\circ} / \mathrm{GHz}$ resolution. Since varying the attenuators also causes the phase shift through them to change slightly, achieving a precise amplitude and phase shift for the third injected signal requires a careful iteration of both parameters. Solid-state preamplifiers are used to overcome losses in the cables and components, and enable driving of the tube close to saturation. The fundamentals are amplified using two identical HD Comm. 19340 amplifiers (0.8-2.5 GHz, 42-dB gain). A ZHL-42-W minicircuits $(10 \mathrm{MHz}-4.2 \mathrm{GHz}$, $30-\mathrm{dB}$ gain) amplifier is used for amplifying the harmonic, while a ZHL-1042J minicircuits (10 MHz-4.2 GHz, 25-dB gain) amplifier is used for the 3IM. These preamplifiers are operated in a backoff mode to prevent significant nonlinear products from contaminating the amplifiers' output. Thus, attenuators are needed to adjust the fundamental frequency power level inputs to the amplifiers. The three signals are then

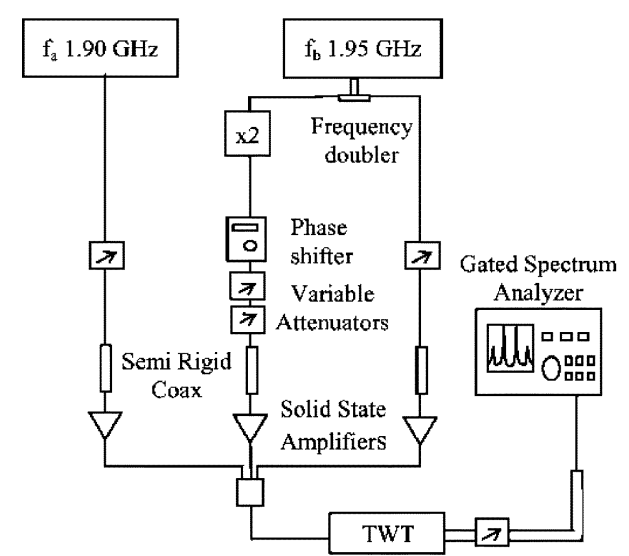

(a)

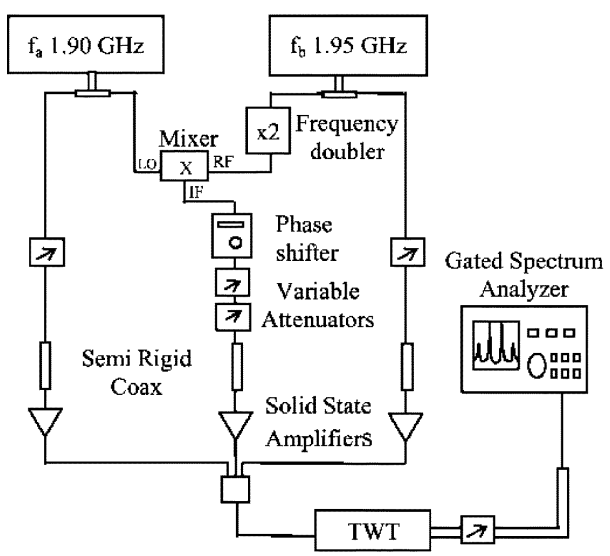

(b)

Fig. 1. Experimental setup for 3IM suppression by direct injection of (a) second harmonic and (b) third-order intermodulation.

combined using a high-power Wilkinson-type combiner from Narda and fed to the XWING input. Since the two fundamental signals will be at much higher power levels than the amount of injected harmonic/3IM needed, isolators (not shown) are used to prevent any fundamental signals from feeding back into the harmonic/3IM paths and disturbing the performance of other components. The input drive to the TWT was set by observing the combiners output on the spectrum analyzer using a two-ft. flexible cable similar to the one that feeds to the XWING input. All spurious frequencies at the TWT input were recorded to be below $30 \mathrm{dBc}(\mathrm{dB}$ below carrier) for all the cases presented in this paper.

The output signal is monitored on an HP 8563EC time-gated spectrum analyzer. The gate delay $(300 \mu \mathrm{s})$ and width $(100 \mu \mathrm{s})$ were chosen to ensure signal capture during a constant voltage section of the $500 \mu$ s cathode voltage pulse. Spectrum analyzer sweep time was set to ensure that the $5-\mathrm{Hz}$ cathode pulse is on at least once for each sample point and the frequency span was adjusted to obtain a fine frequency resolution of $0.1 \mathrm{MHz}$. An attenuator is used at the input to the spectrum analyzer to prevent generation of nonlinear products from the spectrum analyzer's internal mixer that can corrupt the measurements. The shot-toshot variation in power levels was recorded to be $\pm 0.2 \mathrm{~dB}$ for high output powers and increases up to $\pm 0.7 \mathrm{~dB}$ for lower output powers approaching the noise floor. A set of switches is used to select between the sensor signals and the TWT output. Loss in 
the output semi-rigid and switching assembly was also characterized and accounted for.

\section{3IM SUPPRESSION BY SIGNAL INJECTION}

Nonlinearity of the TWT amplifier results in the generation of spurious frequencies at the output of the device. Amongst all intermodulation frequencies $\left(m f_{a} \pm n f_{b}\right)$, the 3IMs $\left(2 f_{b}-f_{a}\right.$, $2 f_{a}-f_{b}$ ) lie closest to the fundamentals. Thus 3IMs are the main contributors to nonlinear distortion and their suppression is most crucial. We investigate two schemes for 3IM suppression using second-order (harmonic) and third-order (3IM) frequency injection.

\section{A. Second-Harmonic Injection}

Second-harmonic injection for 3IM suppression involves injecting the second-harmonics along with the fundamental tones at the TWT input and adjusting their amplitude and phase so as to obtain maximum suppression for the 3IMs. For a twotone case, the injected second-harmonics $\left(2 f_{a}, 2 f_{b}\right)$ beat with the fundamentals $\left(f_{b}, f_{a}\right)$, respectively, to generate additional modes at the 3IM frequencies $\left(2 f_{a}-f_{b}, 2 f_{b}-f_{a}\right)$. The S-MUSE [21] voltage solution at the 3IM frequencies considering dominant modes [17] is given as

$$
\begin{aligned}
V_{3 I M}(z, t)= & \left\{A^{\mathrm{n}[2]} e^{\left(\mu^{\mathrm{n}[2]}+i \kappa^{\mathrm{n}[2]}\right) z}\right. \\
& \left.+A^{\mathrm{n}[3]} e^{\left(\mu^{\mathrm{n}[3]}+i \kappa^{\mathrm{n}[3]}\right) z}\right\} e^{i 2 \pi f_{3 I M}\left(\left(z / u_{0}\right)-t\right)} .
\end{aligned}
$$

3IM suppression by harmonic injection involves cancellation of the natural nonlinearly generated third-order mode $\left[2\left(f_{b}\right)-f_{a}\right.$ or $\left.2\left(f_{a}\right)-f_{b}\right]$ by a second-order mode $\left[\left(2 f_{b}\right)-f_{a}\right.$ or $\left(2 f_{a}\right)-$ $\left.f_{b}\right]$, generated nonlinearly due to the injected harmonic $\left(2 f_{b}\right.$ or $\left.2 f_{a}\right) .{ }^{1}$ The former term grows at a much faster rate (nearly three times the fundamental growth rate) than the latter (about twice the fundamental growth rate) [17], [22]. By injecting a small amount of properly phased harmonic, the nonlinear mode due to the injected harmonic can be made to interfere destructively and cancel the naturally generated nonlinear mode at the output, $z=L$.

Experiments to support this theory were done on the XWING research tube. To simplify the initial setup, only one harmonic $\left(2 f_{b}\right)$ is injected and suppression of the upper 3IM $\left(2 f_{b}-f_{a}\right)$ is shown. However, it should be noted that both 3IMs can be suppressed by simultaneously injecting both second harmonics as discussed later. The XWING has a broad gain-bandwidth of $1.5-6 \mathrm{GHz}$ with a maximum gain of $27 \mathrm{~dB}$ near $3.5 \mathrm{GHz}$ at $15 \mathrm{dBm}$ single-tone input drive. The gain drops to about $15 \mathrm{~dB}$ at the lower and upper limits of the gain bandwidth. The input fundamental frequencies were chosen to lie in the lower part of the gain bandwidth so that the harmonics lie close to

\footnotetext{
${ }^{1}$ When describing modes in this manner we represent a first-order mode of frequency $2 f$ without parentheses, a second-order mode at frequency $2 f$ is written as $2(f)$, and a third-order mode of $f$ is written as $3(f)$. Thus, in this context $2 f$ refers to an injected signal at the second harmonic of frequency $f$, while $2(f)$ refers to the nonlinearly generated second harmonic. Furthermore, sums and differences of frequencies represent formation of nonlinear products. It follows that, for example, $\left(2 f_{a}\right) \pm f_{b}$ are second-order products whereas $2\left(f_{a}\right) \pm f_{b}$ are third-order products.
}

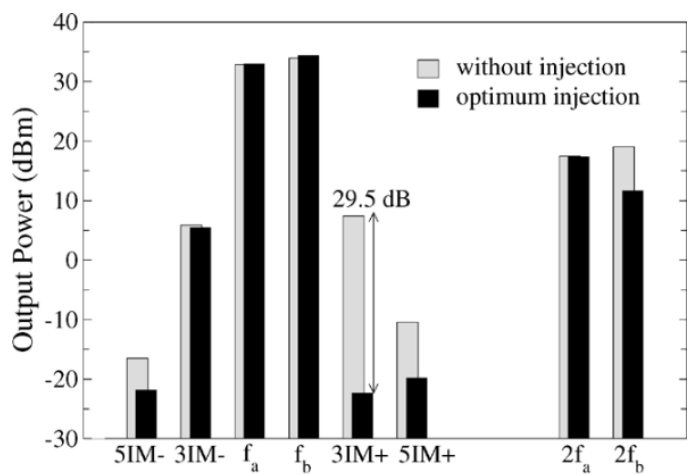

(a)

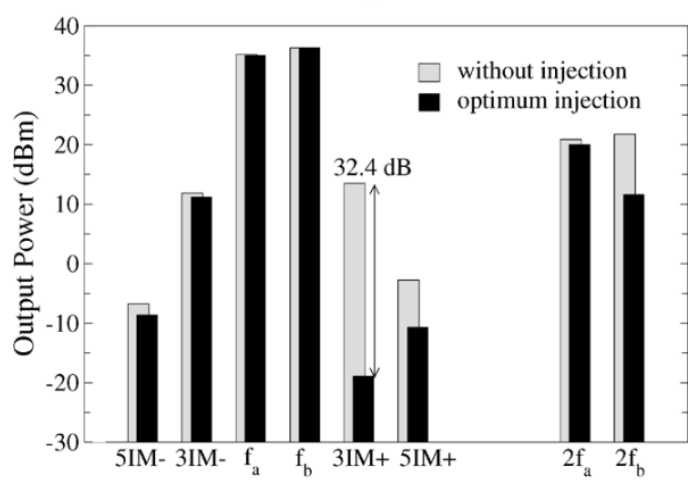

(b)

Fig. 2. Injection of second harmonic of the higher frequency fundamental results in upper 3IM suppression of (a) $29.5 \mathrm{~dB}(56 \mathrm{dBc})$ for $15 \mathrm{dBm} /$ tone drive, and (b) $32.4 \mathrm{~dB}(55.4 \mathrm{dBc})$ for $18 \mathrm{dBm} /$ tone drive.

the gain peak, thus richly incorporating the nonlinear distortion products. Hence, the two input fundamentals were set to 1.90 and $1.95 \mathrm{GHz}$ and the experiment was repeated at drive levels of 15 and $18 \mathrm{dBm} /$ tone. These drive levels correspond to tube operation at the $1 \mathrm{~dB}$ gain compression and near saturation, respectively.

Measurement of output spectra without second-harmonic injection shows the upper 3IM $(2.00 \mathrm{GHz})$ power level to be $26.5 \mathrm{dBc}$ for the $15 \mathrm{dBm} /$ tone case and $23 \mathrm{dBc}$ for $18 \mathrm{dBm} /$ tone. The upper second-harmonic at $3.90 \mathrm{GHz}$ was then injected and adjusted in amplitude and phase iteratively to obtain a maximum upper 3IM suppression of $29.5 \mathrm{~dB}(56 \mathrm{dBc})$ and $32.4 \mathrm{~dB}$ $(55.4 \mathrm{dBc})$ for 15 and $18 \mathrm{dBm} /$ tone, respectively. The injected harmonic power required to obtain maximum suppression was $\sim 17-18 \mathrm{dBc}$ for both the fundamental drive levels. Output spectra without and with injection are shown in Fig. 2 for both cases.

The output spectrum was observed on a gated spectrum analyzer. The error bars on the power levels were recorded to be $\pm 0.2 \mathrm{~dB}$ for fundamentals, $\pm 0.3 \mathrm{~dB}$ for lower 3IM and harmonics, and $\pm 0.5 \mathrm{~dB}$ for the 5IMs. The error bar in the measurement of the upper 3IM power is higher $( \pm 1 \mathrm{~dB})$ with injection since the power level is very close to the noise floor and slight drifts in injected amplitude and phase also cause suppression to change. Nevertheless, the error bars are insignificant relative to the power levels and the degree of suppression obtained.

It can be seen that suppression is more with higher drive power (18 versus $15 \mathrm{dBm}$ ) since the initial level of 3IM without 
TABLE I

MODES GENERATED BY SECOND-HARMONIC INJECTION AT VARIOUS FREQUENCIES OF INTEREST

\begin{tabular}{lll}
\hline Lower 5IM: & $3\left(f_{a}\right)-\left(2 f_{b}\right)$ & fourth-order mode \\
Lower 3IM: & $2\left(f_{a}\right)+\left(f_{b}\right)-\left(2 f_{b}\right)$ & fourth-order mode \\
Upper fundamental: & $\left(2 f_{b}\right)-\left(f_{b}\right)$ & second-order mode \\
Upper 3IM: & $\left(2 f_{b}\right)-\left(f_{a}\right)$ & second-order mode \\
Upper 5IM: & $\left(2 f_{b}\right)+\left(f_{b}\right)-2\left(f_{a}\right)$ & fourth-order mode \\
Upper second-harmonic: & $\left(2 f_{b}\right)$ & first-order (injected) mode
\end{tabular}

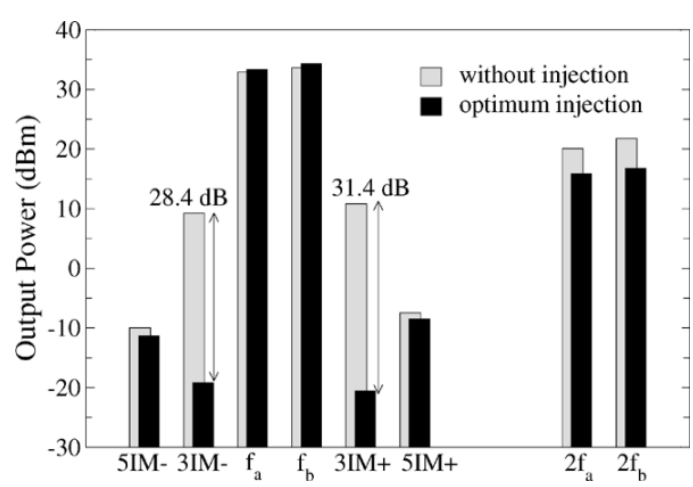

Fig. 3. Both upper and lower 3IMs are suppressed by 28.4 and $31.4 \mathrm{~dB}$ with simultaneous injection of second harmonics of both the fundamentals at $15 \mathrm{dBm} /$ tone.

injection was also higher, while the minimum suppressed power level obtainable is limited by the spectrum analyzers ability to adequately resolve the signals which are close to the noise floor and the small amounts (less than $30 \mathrm{dBc}$ ) of 3IM level present at the TWT input arising from preamplifiers.

It should be noted that optimizing injected harmonic amplitude and phase for 3IM suppression also led to suppression of the upper and lower 5IMs (fifth-order intermodulation frequencies) as well as the injected harmonic frequency. This reaffirms the presence of distinct multiple modes at a given frequency, where the mode due to beating of injected harmonic cancels the nonlinear mode at that frequency depending on the phasing between the two. In this case of upper second-harmonic injection, the modes at each of these frequencies due to the injected harmonic $2 f_{b}$ can be shown as Table I.

Thus, the injected amplitude and phase can be optimized to either achieve maximum suppression at a particular frequency or to obtain some desired suppression at multiple frequencies. ([18] shows results for harmonic injection optimized for secondharmonic suppression.)

While only suppression of the upper 3IM is demonstrated in the previous experiment, it is possible to suppress both upper and lower 3IMs by simultaneous injection of both second-harmonics. Experimental measurements were taken for the same input frequencies of 1.90 and $1.95 \mathrm{GHz}$ at $15 \mathrm{dBm} /$ tone drive. Optimized injection of harmonics at 3.80 and $3.90 \mathrm{GHz}$ resulted in 28.4 and $31.4 \mathrm{~dB}$ of suppression, respectively, for the lower and upper 3IMs at the output, as shown in Fig. 3.

Suppression at the lower 3IM is $3 \mathrm{~dB}$ less than for the upper 3IM, which is expected since the tube gain is less at the lower frequency. Again, it is observed that both harmonics are suppressed by $4-5 \mathrm{~dB}$ in addition to the 3IMs. However, the amount of suppression for the 5IMs is reduced. This reduced suppression at the 5IMs is explainable by observing that with the injection of both harmonics, there are many more modes that contribute to the total solution at each of these frequencies, e.g., at the upper 5IM $\left(3 f_{b}-2 f_{a}\right)$, apart from the fourth-order mode $\left(2 f_{2}\right)+\left(f_{2}\right)-2\left(f_{1}\right)$ present with injection of $2 f_{2}$ only, now there is a third-order mode $\left(2 f_{2}\right)+\left(f_{2}\right)-\left(2 f_{1}\right)$ due to the presence of injected $2 f_{1}$.

Thus, it is verified that both the 3IMs can be suppressed by injecting the corresponding harmonics. However, for simplicity of set-up and equipment limitations, the rest of the experimental investigation focuses on suppression of the upper 3IM.

\section{B. 3IM Injection}

Injection of a second-harmonic signal to suppress the 3IM frequency suggests the more obvious scheme of directly injecting third-order distortion products, such as the 3IM frequency itself. In fact, the conventional predistortion linearizer works on this principle and can be thought of as a generator of 3IM products whose amplitudes and phases are adjusted according to an inverse transfer characteristic to cancel the nonlinearly generated 3IMs. Thus, it is insightful to investigate this scheme and compare with second-harmonic injection for 3IM suppression.

For a two-tone input at $f_{a}, f_{b}$ with injection of one or both 3IMs at $2 f_{b}-f_{a}$ and/or $2 f_{a}-f_{b}$, the S-MUSE solution at the 3IM frequencies is given as

$$
\begin{aligned}
V_{3 I M}(z, t)= & \left\{A^{\mathrm{dr}} e^{\left(\mu^{\mathrm{dr}}+i \kappa^{\mathrm{dr}}\right) z}\right. \\
& \left.+A^{\mathrm{nl}[3]} e^{\left(\mu^{\mathrm{n}[3]}+i \kappa^{\mathrm{nl}[3]}\right) z}\right\} e^{i 2 \pi f_{3 I M}\left(\left(z / u_{0}\right)-t\right)} .
\end{aligned}
$$

3IM injection for 3IM suppression involves cancellation of the natural nonlinearly generated third-order mode $\left[2\left(f_{b}\right)-f_{a}\right.$ or $\left.2\left(f_{a}\right)-f_{b}\right]$ by a first-order injected mode $\left[2 f_{b}-f_{a}\right.$ or $2 f_{a}-$ $f_{b}$ ] due to the injected signal at the 3IM frequency. Again, the growth rate of the third-order natural mode (approximately three times the fundamental) is greater than for the first-order driven mode (nearly the same as the fundamental). A small amount of properly phased signal injected at the 3IM frequency can cancel the naturally generated nonlinear mode at the output, $z=L$, by destructive interference.

Experimental measurements on XWING were taken for the same set of frequencies and power levels as for harmonic injection. The two input fundamentals were set to 1.90 and $1.95 \mathrm{GHz}$. The experiment was repeated at the two drive levels of 15 and $18 \mathrm{dBm} /$ tone. Suppression of only the upper 3IM $\left(2 f_{b}-f_{a}\right)$ is targeted here. However, both 3IMs can be suppressed by injecting two signals at each of the 3IM frequencies as discussed in the previous section. Measurement of output spectra without injection shows the upper 3IM $(2.00 \mathrm{GHz})$ power level without injection of the second-harmonic to be $26.5 \mathrm{dBc}$ for the $15 \mathrm{dBm} /$ tone case and $23 \mathrm{dBc}$ for $18 \mathrm{dBm} /$ tone. The upper 3IM at $2.00 \mathrm{GHz}$ was then injected and adjusted in amplitude and phase iteratively to obtain a maximum upper 3IM suppression of $27 \mathrm{~dB}(53.5 \mathrm{dBc})$ and $30 \mathrm{~dB}(53 \mathrm{dBc})$ for fundamental power levels of 15 and $18 \mathrm{dBm} /$ tone, respectively. The injected 3IM 


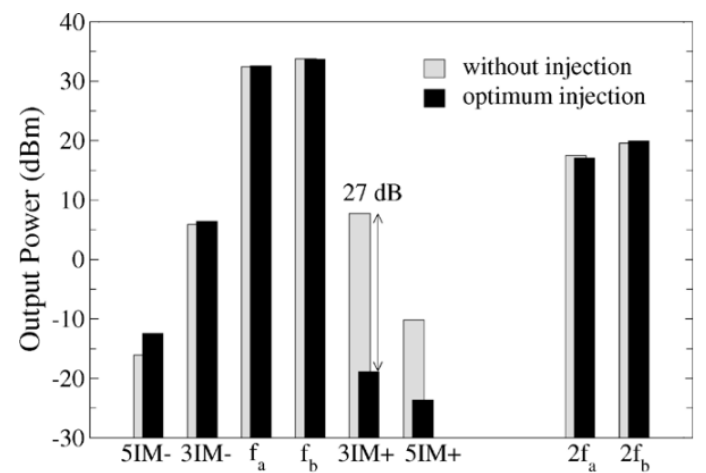

(a)

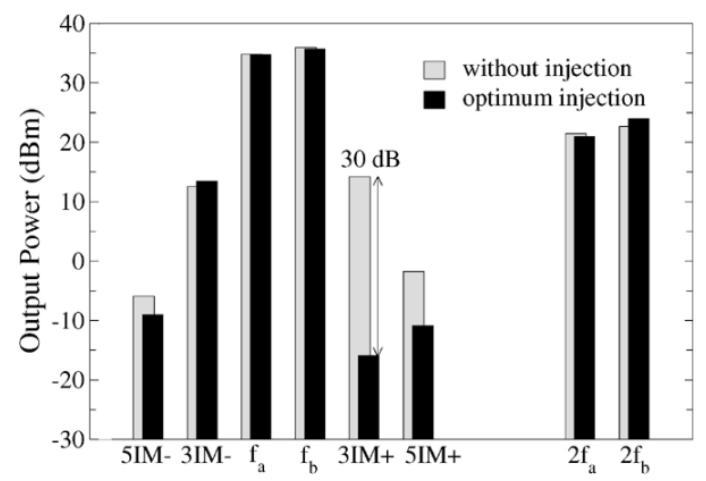

(b)

Fig. 4. Injection of a signal at the upper 3IM frequency results in upper 3IM suppression of (a) $27 \mathrm{~dB}(53.5 \mathrm{dBc})$ for $15 \mathrm{dBm} /$ tone drive, and (b) $30 \mathrm{~dB}$ $(53 \mathrm{dBc})$ for $18 \mathrm{dBm} /$ tone drive.

TABLE II

MODES GENERATED BY 3IM INJECTION AT VARIOUS FREQUENCIES OF INTEREST

\begin{tabular}{lll}
\hline Lower 5IM: & $2\left(f_{a}\right)-\left(2 f_{b}-f_{a}\right)$ & third-order mode \\
Upper 3IM: & $\left(2 f_{b}-f_{a}\right)$ & first-order (injected) mode \\
Upper 5IM: & $\left(2 f_{b}-f_{a}\right)+\left(f_{b}\right)-\left(f_{a}\right)$ & third-order mode \\
Upper second-harmonic: & $\left(2 f_{b}-f_{a}\right)+\left(f_{a}\right)$ & second-order mode
\end{tabular}

power required was comparable to the harmonic injection case ( $17 \mathrm{dBc}$ ). Output spectra without and with injection are shown in Fig. 4 for both cases. The suppression is again found to be more with a higher drive level as is the case for harmonic injection, due to the higher 3IM level present prior to injection.

For 3IM injection, the upper 5IM is also suppressed in addition to the upper 3IM by $13.5 \mathrm{~dB}$ for $15 \mathrm{dBm} /$ tone drive and by $9 \mathrm{~dB}$ for $18 \mathrm{dBm} /$ tone drive. However, it should be noted that none of the second-harmonics are suppressed. Further, the lower 5IM level is actually found to be $4 \mathrm{~dB}$ higher with injection than without injection for the $15 \mathrm{dBm} /$ tone case, as is the upper second-harmonic for the $18 \mathrm{dBm} /$ tone case. This destructive and constructive cancellation at the various frequencies is explainable since there is a mode at each of these frequencies formed by beating of the injected 3IM signal as shown in Table II.

The phase relationship between the modes due to the injected signal and the naturally generated modes determines the nature of the effect-destructive or constructive. In general, it has been found [4] that predistortion equalizers, which work by injecting
3IMs, cause the higher order intermods to increase. This is observed experimentally for the lower 5IM for the $15 \mathrm{dBm} /$ tone case as shown in Fig. 4.

\section{Spatial Evolution}

Measurements of the wave spectrum along the tube were taken to reveal the destructive and constructive effects of the two interfering modes. Since the modes have different growth rates and wavelengths, injected amplitude and phase can be optimized to give suppression at a single axial location. It has been shown in [18] that this location of maximum suppression can be shifted along the axis by changing the input parameters.

The sensors along the helix in the XWING TWT enable measurement of the spatial evolution of the wave spectrum. This was done for input frequencies of 1.90 and $1.95 \mathrm{GHz}$ at $15 \mathrm{dBm} /$ tone drive by injecting both second-harmonics (3.80 and $3.90 \mathrm{GHz}$ ) and optimizing their amplitude and phase for maximum suppression of 28.4 and $31.4 \mathrm{~dB}$ for the respective 3IMs at the output. Fig. 5 shows the spectrum measurements at the input, sensor 1 (which is located halfway through the tube), sensor 4 (located close to the output port) and at the output.

The input spectra shows that the optimum injected harmonic powers required for obtaining suppression are $-3.3 \mathrm{dBm}$ for $3.80 \mathrm{GHz}$ and $-2.1 \mathrm{dBm}$ for $3.90 \mathrm{GHz}$. Thus, very small amounts (less than $17 \mathrm{dBc}$ ) of properly phased harmonics are needed to obtain up to $30 \mathrm{~dB}$ of 3IM suppression at the output. The 3IM level at the input is less than $30 \mathrm{dBc}$ and is due to the preamplifiers. It can be seen that the 3IM levels obtained after suppression at the output are lower than the initial level of 3IM present at the input. While all the frequencies show almost monotone growth along the axis without injection, presence of the injected mode causes destructive and constructive effects along the axis.

The significant difference in the power levels of the 3IMs between sensor 4 (which is located close to the output) and the output suggests that suppression is quite sensitive to injected amplitude and phase. The sensitivity of the suppression to injected amplitude and phase is investigated in the next section.

\section{SENSITIVITy ANALYSIS FOR SignAl INJECTION}

While the experimental results presented in previous sections show that the signal injection techniques can provide $>50 \mathrm{dBc}$ of suppression for the targeted nonlinear distortion product, the amount of suppression obtained is sensitive to the injected signals amplitude and phase. This requires fine tuning of these two parameters as well as maintaining stability to phase drifts. Thus, it is instructive to determine the required tolerance for these parameters to maintain a desired amount of suppression.

Experimental measurements were taken to study the sensitivity of the suppression to injected amplitude and phase for upper second-harmonic injection with fundamental frequencies of 1.90 and $1.95 \mathrm{GHz}$ at $18 \mathrm{dBm} /$ tone. Since changing the injected amplitude by adjusting the attenuator causes the phase to also change, a phase sweep was done at each injected amplitude level.

As can be seen from the contour plot of Fig. 6, the amount of suppression obtained is quite sensitive to the precision of 


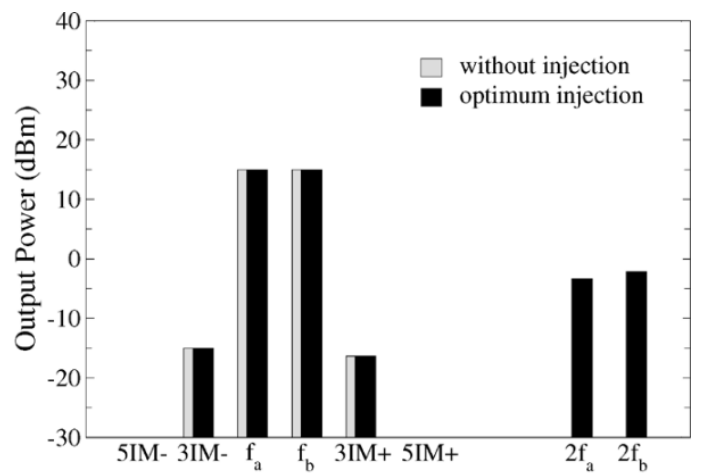

(a)

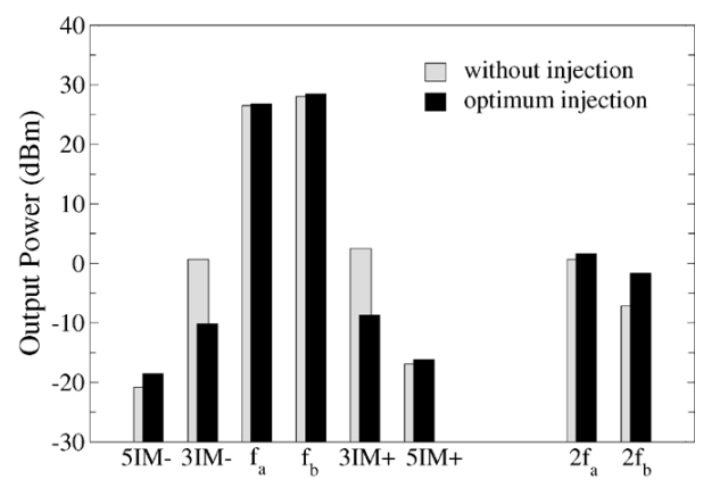

(b)

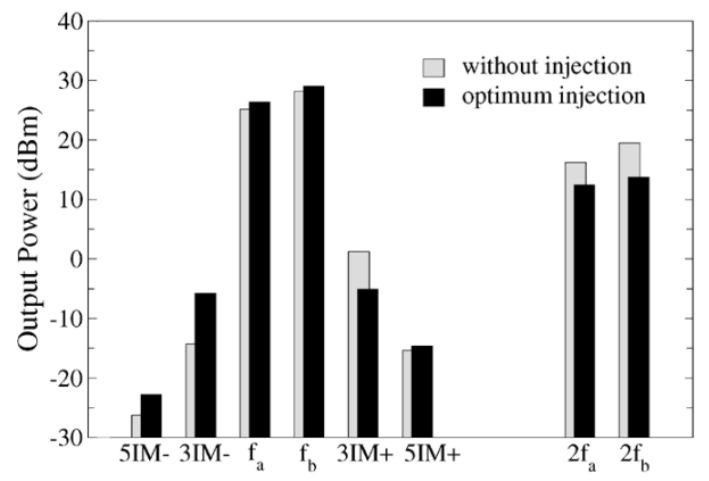

(c)

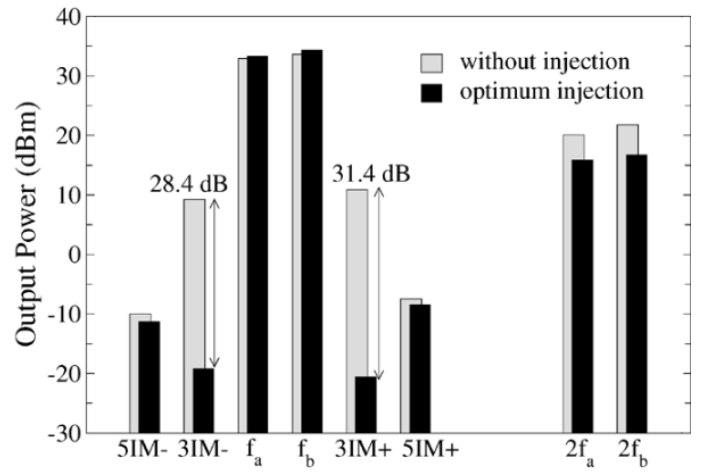

(d)

Fig. 5. Spatial evolution of the wave spectrum with and without injection at (a) the input port, (b) sensor 1 (located halfway through the tube), (c) sensor 4 (located close to the output port), and (d) output port.

the injected amplitude and phase. Nevertheless, a suppression of greater than $20 \mathrm{~dB}$ is obtained for simultaneous variations of $\pm 0.8 \mathrm{~dB}$ in injected amplitude and $\pm 5^{\circ}$ in injected phase. These tolerances are readily achieved in practice. Further suppression

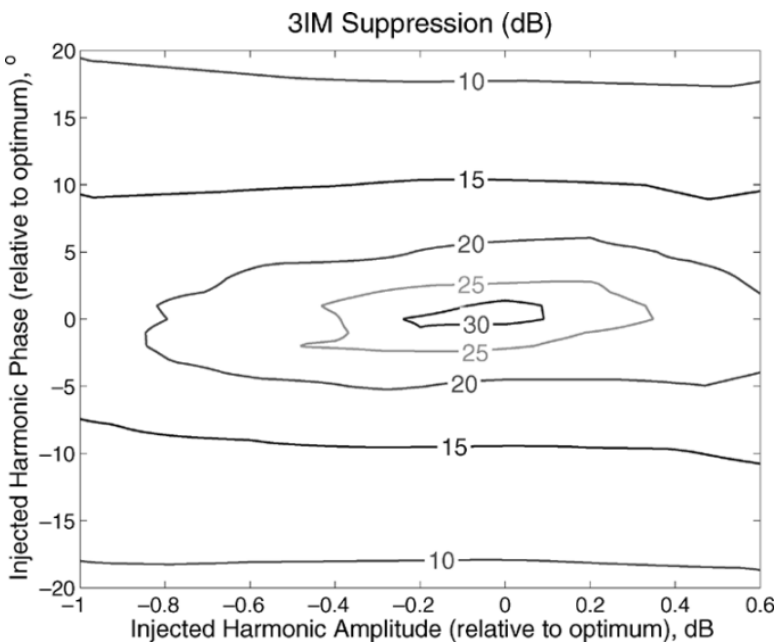

Fig. 6. Sensitivity of 3IM suppression by harmonic injection to injected amplitude and phase.

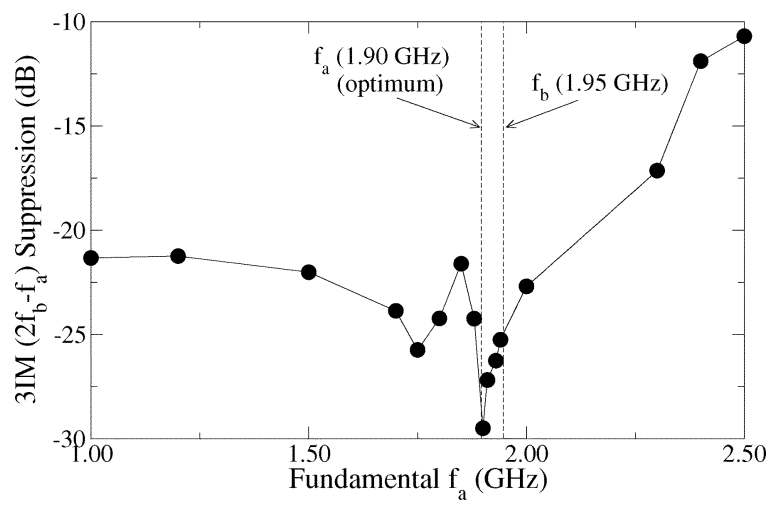

Fig. 7. 3IM suppression of more than $20 \mathrm{~dB}$ is maintained when varying the frequency of fundamental $f_{a}$ over $0.9 \mathrm{GHz}$ after optimizing harmonic injection for $f_{a}=1.90 \mathrm{GHz}$. Thus, suppression is effective over a wide bandwidth.

depends on fine tuning of these parameters. Sensitivity measurements with other signal injection schemes also exhibited similar behavior.

\section{BANDWIDTH OF SUPPRESSION BY SIGNAL INJECTION}

Along with amplitude and phase sensitivity, it is also instructive to determine the bandwidth over which suppression is effective. A higher bandwidth is desired since it implies that suppression is relatively insensitive to changes in the modulation frequency.

Experimental measurement of fundamental frequency sensitivity of 3IM suppression was done for the second harmonic injection scheme by varying the frequency $f_{a}$ and keeping $f_{b}$ fixed, and injecting $2 f_{b}$ to suppress the 3IM, $2 f_{b}-f_{a}$. With fundamental frequencies of 1.90 and $1.95 \mathrm{GHz}$ at $15 \mathrm{dBm} /$ tone, a $3.90-\mathrm{GHz}$ harmonic was injected and optimized to obtain a maximum suppression of $29.5 \mathrm{~dB}$ for the $3 \mathrm{IM}$ at $2.00 \mathrm{GHz}$. The frequency of the fundamental at $1.90 \mathrm{GHz}$ was then varied over a wide range and the suppression observed as shown in Fig. 7.

Here, $f_{a}$ can be thought of as a modulation over $f_{b}$. It can be seen that although maximum suppression is obtained at the optimization frequency, the overall bandwidth of effective suppression is quite large. A 3IM suppression of $>20 \mathrm{~dB}$ is possible 
over a $0.9 \mathrm{GHz}$ change in the lower fundamental frequency $f_{a}$ ( $47 \%$ relative bandwidth). We notice an asymmetry in the suppression obtained as $f_{a}$ becomes greater than $f_{b}$ and the 3IM frequency of interest $2 f_{b}-f_{a}$ now becomes the lower 3IM (rather than upper 3IM, when $f_{a}$ was less than $f_{b}$ ). Currently we are not able to explain this experimental observation. However, practical bandwidths are in the $\mathrm{MHz}$ range, for which suppression is substantial as seen in Fig. 7.

The high bandwidth of suppression also supports the notion that the nonlinearly generated second-harmonic without injection is mainly responsible for generating intermodulation distortion products [24]. While the suppression is quite sensitive to injected harmonic amplitude and phase, as long as the injected second-harmonic amplitude and phase remains fixed, the suppression is relatively insensitive to shifts in the fundamental frequency it beats with to form the intermodulation distortion products.

\section{COMPARISON AND SUMMARY}

This paper provided a detailed experimental investigation of signal injection technique for nonlinear distortion suppression in TWTs and an improved understanding of second-order versus third-order signal injection or predistortion. While third-order (3IM) predistortion is widely being used for TWT linearization, the results presented in this paper indicated that second-order predistorters may be even more effective, especially for wideband TWTs.

It was observed that for the 3IM injection scheme, minimizing power at the third-order intermods results in increase in power at some higher order intermods (Fig. 4). This is also a general observation for predistortion equalizers that work on the principle of 3IM injection [4]. On the other hand, our harmonic injection results show suppression of higher order intermods as well as the harmonics, along with the targeted 3IM signal as seen in Fig. 2. This is desired for communication applications since, although the desire is to target the third-order intermods (the intermodulation products closest to the fundamentals), the overall spurious frequency content also needs to be low.

Our observations suggest that second harmonic injection may be superior to 3IM injection with regard to exacerbation of 5IM products. Specifically, our data indicate that second-harmonic injection only modifies the 5IM products by modest amounts ( $\sim 8 \mathrm{~dB})$, and in a favorable manner (i.e., the 5IM amplitude with injection is less than the 5IM amplitude without injection). In contrast, 3IM injection can modify the 5IM products by more significant levels $(\sim 12 \mathrm{~dB})$, and in many instances in a deleterious manner (i.e., the 5IM amplitude with injection is larger than the 5IM amplitude without injection). While we do not have a definitive explanation for this difference, it is consistent with the fact that nonlinear distortions in TWTs arise as a hierarchical cascade of second-order nonlinearities in the ballistic bunching process [22], e.g., from the $\rho v$ product in the continuity equation. Therefore, third-order products require the pre-existence of second-order products, fifth-order products require the pre-existence of fourth-order products and so on. Thus, one typically observes that $\left|V_{1}\right|>\left|V_{2}\right|>\cdots>\left|V_{m-1}\right|>$
$\left|V_{m}\right|>\cdots$, where $m$ denotes the order of the nonlinear product. The modes due to the injected signal at lower and upper 5IMs are fourth-order modes for second-harmonic injection [ $3\left(f_{a}\right)-$ $\left.\left(2 f_{b}\right),\left(2 f_{b}\right)+\left(f_{b}\right)-2\left(f_{a}\right)\right]$ and third-order modes for 3IM injection $\left[2\left(f_{a}\right)-\left(2 f_{b}-f_{a}\right),\left(2 f_{b}-f_{a}\right)+\left(f_{b}\right)-\left(f_{a}\right)\right]$. Since a higher order mode has a smaller amplitude than a lower-order mode, second harmonic injection is not expected to exacerbate the higher order intermodulation frequencies as much as 3IM injection.

To conclude, this paper presented an improved understanding of the nonlinear phenomena involved in TWT linearization via second and third-order signal injection or predistortion. Results showed that signal injection schemes provided a high 3IM suppression of greater than $55 \mathrm{dBc}$ with our readily available laboratory components, which is close to the commercially desired value of $60 \mathrm{dBc}$ [25] in many applications. Sensitivity analysis of the scheme shows that a suppression of $20 \mathrm{~dB}$, leading to a 3IM level of $45 \mathrm{dBc}$, is maintained for a simultaneous change of $\pm 0.8 \mathrm{~dB}$ in injected amplitude and $\pm 5^{\circ}$ in injected phase. Also, the suppression is effective over a large frequency bandwidth. While comparable suppression can be obtained by either second or third-order injection, the results presented in this paper indicate that second-order predistortion should have certain advantages over conventional third-order predistortion for wide-band TWTs. These include simultaneous suppression of second-harmonic and reduced exacerbation of 5IM products.

\section{REFERENCES}

[1] M. Kumar, J. C. Whartenby, and H. J. Wolkstein, "GaAs dual-gate FET linearizer for traveling-wave tube amplifiers," Microw. J., vol. 27, no. 8, pp. 127-128 130, Aug. 1984.

[2] A. N. D’Andrea, V. Lottici, and R. Reggiannini, "RF power amplifier linearzation through amplitude and phase predistortion," IEEE Trans. Commun., vol. 44, no. 11, pp. 1477-1484, Nov. 1996.

[3] D. M. Goebel, R. R. Liou, W. L. Menninger, X. Zhai, and E. A. Adler, "Development of linear traveling wave tubes for telecommunications applications," IEEE Trans. Electron Devices, vol. 48, no. 1, pp. 74-81, Jan. 2001.

[4] A. Katz, "Linearization: Reducing distortion in power amplifiers," IEEE Microw. Mag., vol. 2, no. 4, pp. 37-49, Dec. 2001.

[5] O. Shimbo, "Effects of intermodulation, AM-PM conversion, and additive noise in multicarrier TWT systems," Proc. IEEE, vol. 59, pp. 230-238, 1971.

[6] H.-M. Park, D.-H. Baek, K.-I. Jeon, and S. Hong, "A predistortion linearizer using envelope-feedback technique with simplified carrier cancellation scheme for class-A and class-AB power amplifiers,' IEEE Trans. Microw. Theory Tech., vol. 48, no. 6, pp. 898-904, Jun. 2000.

[7] T. Chen, Y. Goren, C. Jensen, P. Lally, and D. Gagne, "A novel technology for linearizing traveling wave tube amplifiers," in Proc. IEEE MTT-S Int. Microwave Symp., vol. 2, 2002, pp. 773-776.

[8] D. C. Cox, "Linear amplification with nonlinear components," IEEE Trans. Commun., no. 12, pp. 1942-1945, Dec. 1974.

[9] A. Choffrut, B. D. Van Veen, and J. H. Booske, "Traveling wave tubebased LINC transmitters," IEEE Trans. Electron Devices, vol. 50, no. 5, pp. 1405-1407, May 2003.

[10] J. J. Hamilton and D. Zavadil, "Harmonically-enhanced two-octave TWTA," Microw. J., vol. 15, pp. 24-25, 1972.

[11] W. E. Garrigus and M. L. Glick, "Multi-octave high-power TWT operation," Microw. J., vol. 18, pp. 35-40, 1975.

[12] J. T. Mendel, "Helix and coupled-cavity traveling-wave tubes," Proc. IEEE, vol. 61, no. 3, pp. 280-288, Mar. 1973.

[13] N. J. Dionne, "Harmonic generation in octave bandwidth traveling-wave tubes," IEEE Trans. Electron Devices, vol. 17, no. 4, pp. 365-372, Apr. 1970 . 
[14] S. K. Datta, P. K. Jain, and B. N. Basu, "Control of IM3 distortion in helix TWTs by harmonic injection - An Eulerian hydrodynamical study," IEEE Trans. Electron Devices, vol. 48, no. 1, pp. 62-66, Jan. 2001.

[15] S. K. Datta, P. K. Jain, M. D. Rajnarayan, and B. N. Basu, "Eulerian analysis for harmonic generation in a helix travelling-wave tube," Int. J. Electron., vol. 85, no. 3, pp. 377-395, 1998.

[16] J. G. Wöhlbier, "Nonlinear distortion and suppression in traveling wave tubes - Insights and methods," Ph.D. dissertation, Univ. Wisconsin, Madison, WI, 2003.

[17] J. G. Wöhlbier, J. H. Booske, and I. Dobson, "On the physics of harmonic injection in a traveling wave tube," IEEE Trans. Plasma Sci., vol. 32, no. 3, pp. 1073-1085, Jun. 2004.

[18] A. Singh, J. G. Wöhlbier, J. H. Booske, and J. E. Scharer, "Experimental verification of the mechanisms for nonlinear harmonic growth and suppression by harmonic injection in a traveling wave tube," Phys. Rev. Lett., vol. 92, 2004.

[19] O. Sauseng, M. A. Huisjen, and W. E. Garrigus, "Reduction of intermodulation distortion with harmonic injection for wide-band traveling-wave tubes," in IEDM Tech. Dig., 1975, pp. 411-414.

[20] M. A. Wirth, A. Singh, J. E. Scharer, and J. H. Booske, "Third-order intermodulation reduction by harmonic injection in a TWT amplifier," IEEE Trans. Electron Devices, vol. 49, no. 11, pp. 1082-1084, Nov. 2002.

[21] J. G. Wöhlbier, J. H. Booske, and I. Dobson, "The multifrequency spectral Eulerian (MUSE) model of a traveling wave tube," IEEE Trans. Plasma Sci., vol. 30, no. 3, pp. 1063-1075, Jun. 2002.

[22] J. G. Wöhlbier, I. Dobson, and J. H. Booske, "Generation and growth rates of nonlinear distortions in a traveling wave tube," Phys. Rev. E, Stat. Phys. Plasmas Fluids Relat., vol. 66, 2002.

[23] A. Singh, "Experimental investigation of TWT nonlinearities and distortion suppression by signal injection," M.S. thesis, Univ. Wisconsin, Madison, WI, 2003.

[24] J. G. Wöhlbier and J. H. Booske, "Mechanisms for phase distortion in a traveling wave tube," Phys. Rev E, Stat. Phys. Plasmas Fluids Relat., vol. 69, 2004.

[25] D. J. Jennings, A. Bateman, and J. P. McGeehan, "Adjacent channel power and error-vector magnitude performance of reduced complexity CALLUM systems," Proc. Inst. Elect. Eng., Comm., vol. 146, no. 5, pp. 297-302, Oct. 1999.

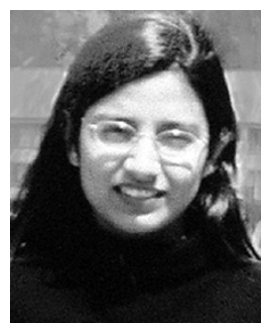

Aarti Singh received the B.E. degree in electronics and communication engineering from the Netaji Subhas Institute of Technology, Delhi, India in 2001 and the M.S. degree in electrical engineering from the University of Wisconsin, Madison, in 2003, where she is currently pursuing the Ph.D. degree.

Her research interests span the field of communications including RF, wireless, and networking issues. She has been involved with experimental work on linearization schemes for microwave amplifiers, particularly TWTs. Currently, she is working on smart antenna systems for wireless ad hoc networks.

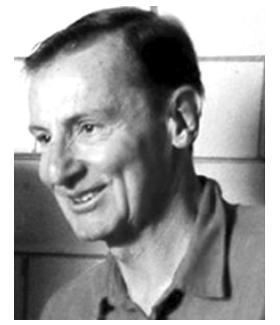

John E. Scharer (SM'90) received the B.S., M.S., and $\mathrm{Ph} . \mathrm{D}$. degrees in electrical engineering from the University of California, Berkeley.

$\mathrm{He}$ is a Professor in the Department of Electrical and Computer Engineering and Co-Director of the Center for Plasma Theory and Computation, University of Wisconsin, Madison. He has spent sabbaticals at the CEA Fontenary-aux-Roses, France, in 1970, at the JET tokamak at Culham, U.K., in 1983, and at the Australian National University, Sydney, in 2000 working on RF plasma physics. He has substantial cumulative research experience in microwave vacuum electronics, excimer laser plasma creation, optical spectroscopy, radiofrequency plasma sustainment, microwave and millimeter-wave diagnostics and the chemistry of organic seed gas plasmas in air. He also has extensive experience in theoretical, computational, and experimental research on antenna coupling and wave propagation, heating and creative diagnostics in plasmas and vacuum electronics.

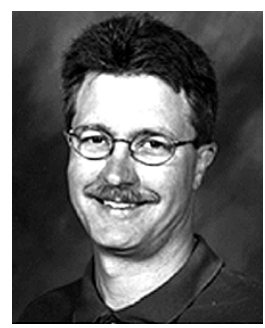

John H. Booske (SM'93) received the Ph.D. degree in nuclear engineering from the University of Michigan, Ann Arbor, in 1985.

He was a Research Scientist with the University of Maryland, College Park, from 1985 to 1989. In 1990, he joined the faculty of the University of Wisconsin, Madison, where he is a Professor of electrical and computer engineering. In 2001, he was appointed $\mathrm{Di}$ rector of the Materials Science Program. His research interests include the experimental and theoretical study of coherent electromagnetic radiation sources and their applications, with emphasis in the RF, microwave, millimeter-wave, and tetrahertz regimes. Recent research activities include vacuum electronics, microwave and RF heating of materials, and biological/biomedical applications of microwave and RF fields. He is coeditor of the book Microwave and Radio Frequency Applications (Westerville, OH: American Ceramic Society, 2003) and coeditor of the book Microwave and Millimeter-Wave Power Electronics (Piscataway, NJ: IEEE Press, 2004).

Dr. Booske has received the University of Wisconsin Vilas Associate Faculty Award for research excellence and the U.S. National Science Foundation Presidential Young Investigator Award. He has been honored with many teaching awards, including the University of Wisconsin Chancellors Distinguished Teaching Award, and the Benjamin Smith Reynolds Award for Excellence in Teaching Engineering. He served as Co-Director of a U.S. DoD MURI99 Consortium on Innovative Microwave Vacuum Electronics, and as Director of a MURI04 Consortium on cathode field emission and RF window breakdown in high-power microwave sources. He has been a Guest Editor of the IEEE TRANSACTIONS ON Plasma SCIENCE.

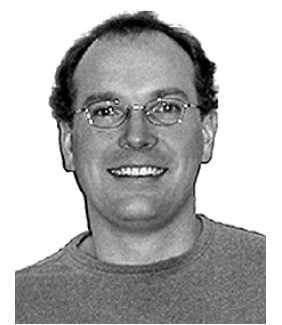

John G. Wöhlbier received the Ph.D. degree in electrical engineering from the University of Wisconsin, Madison, in 2003.

In the summer of 2003 he was an Engineering Physics Postdoctoral Scientist at the University of Wisconsin where he worked on ballooning instabilities in three-dimensional plasmas. In 2004, he worked on vacuum device physics as an Agnew National Security Postdoctoral Fellow, Los Alamos National Laboratory. Currently, he is working on the Advanced Simulation and Computing program (ASC) in the Continuum Dynamics group (CCS-2) at Los Alamos. His research interests include plasma physics, fluid dynamics, computational physics, dynamical systems, vacuum electronics, and applied math. 